\title{
Cupcake adicionado de farinha de resíduos de brócolis: análise físico-química e sensorial entre crianças
}

\section{Cupcake added to broccoli flour: physicochemical and sensory analysis among children}

Cupcake añadido de harina de residuos de brócoli: análisis físico-químico y sensorialentre niños

\author{
Ana Júlia Machado Coutinho ${ }^{1}$ \\ Mirelly Marques Romeiro Santos ${ }^{2}$ \\ Elisvânia Freitas dos Santos ${ }^{3}$ \\ Daiana Novello ${ }^{4}$
}

${ }^{1}$ Graduada em Nutrição pela Universidade Estadual do Centro-Oeste (UNICENTRO). E-mail: anajuucou@gmail.com, ORCID: http://orcid.org/0000-0002-6320-0443

${ }^{2}$ Doutoranda do Programa de Pós-Graduação em Saúde e Desenvolvimento na Região Centro-Oeste na Universidade Federal de Mato Grosso do Sul (UFMS). Mestre em Saúde e Desenvolvimento na Região Centro-Oeste pela UFMS. Graduada em Nutrição pela UFMS. E-mail: mirellymrsantos@gmail.com, ORCID: http://orcid.org/0000-0002-1024-1540

${ }^{3}$ Doutora em Cirurgia Experimental pela Universidade Estadual de Campinas (UNICAMP). Mestre em Alimentos e Nutrição pela Faculdade de Engenharia de Alimentos da UNICAMP. Professora do Curso de Nutrição da Faculdade de

Ciências Farmacêuticas, Alimentos e Nutrição (FACFAN) pela Universidade

Federal de Mato Grosso do Sul (UFMS). E-mail: elisvania@gmail.com, ORCID: http://orcid.org/0000-0002-1528-6035

${ }^{4}$ Doutora em Tecnologia de Alimentos pela Universidade Estadual de Campinas (UNICAMP). Mestre em Ciências Veterinárias pela Universidade Federal do Paraná (UFPR). Professora do Curso de Nutrição da Universidade

Estadual do Centro-Oeste (UNICENTRO). E-mail: nutridai@gmail.com, ORCID: http://orcid.org/0000-0003-0762-5292 
Resumo: $\mathrm{O}$ objetivo do estudo foi avaliar a aceitabilidade sensorial de cupcake adicionado de diferentes níveis de Farinha de Resíduos de Brócolis (FRB). Também, determinar a composição físico-química da formulação padrão e daquela contendo maior teor de FRB com aceitação semelhante a padrão. Foram desenvolvidas cinco formulações de cupcake: F1 (0\% de FRB) e as demais adicionadas de 3\% (F2), 6\% (F3), 9\% (F4) e 12\% (F5) de FRB. Participaram da avaliação sensorial 83 julgadores não treinados de 7 a 10 anos. Maiores notas $(p<0,05)$ foram verificadas para as amostras F1, F2 e F3 em todos os atributos e para a aceitação global, sem diferença entre si. As amostras F4 e F5 foram aquelas menos aceitas, com notas similares $(p>0,05)$. Maiores teores de cinzas e de fibra alimentar foram verificados em F3. Não houve alteração $(p>0,05)$ nos conteúdos de proteína, lipídio, carboidrato e energia entre as amostras F1 e F3. Conclui-se que um nível de adição de até $6 \%$ de FRB em cupcake melhora o perfil nutricional do produto. Além disso, apresenta aceitabilidade semelhante ao produto padrão, com boas expectativas de comercialização.

Palavras chave: nutrientes; fibras na dieta; aproveitamento integral dos alimentos.

Abstract: This paper's purpose was to evaluate the sensory acceptability of cupcake made with different amounts of Broccoli Residues Flour (BRF). It is also intended to determine the physicochemical composition of the standard formulation and of that one containing higher BRF content, with the most similar acceptance to standard. We developed five cupcake formulations: F1 (O\% BRF) and the following ones, F2 (3\%), F3 (6\%), F4 (9\%), F5 (12\%) of BRF. A total of 83 untrained judges aged 7 to 10 years participated in the sensory evaluation. Higher scores $(p<0.05)$ were verified for $F 1, F 2$, and F3 samples in all attributes and for global acceptance, with no difference between them. Samples F4 and F5 were the least accepted, with similar scores ( $p>0.05)$. Higher levels of ash and dietary fiber were observed in F3. There was no change ( $p>0.05)$ in the protein, lipid, carbohydrate, and energy contents between samples F1 and F3. It was concluded that an addition level of up to $6 \%$ of BRF in cupcake improves the nutritional profile of the product. In addition, it presents similar acceptability to the standard product, with good marketing expectations.

Keywords: nutrients; dietary fibers; whole utilization of foods.

Resumen: El objetivo del estudio fue evaluar la aceptabilidad sensorial de cupcake agregado de diferentes niveles de Harina de Residuos de Brócoli (HRB). También, determinar la composición físico-química de la formulación estándar y de aquella conteniendo mayor contenido de HRB con aceptación semejante a la estándar. Se desarrollaron cinco formulaciones de cupcake: F1 (0\% de HRB) y las demás agregadas de 3\% (F2), 6\% (F3), 9\% (F4) y 12\% (F5) de HRB. Participaron de la evaluación sensorial 83 jueces no entrenados de 7 a 10 años. Las mayores notas $(p<0,05)$ se verificaron para las muestras F1, F2 y F3 en todos los atributos y para la aceptación global, sin diferencia entre sí. Las muestras F4 y F5 fueron aquellas menos aceptadas, con notas similares $(p>0,05)$. Los mayores niveles de cenizas y de fibra alimentaria se verificaron en $F 3$. No hubo alteración $(p>0,05)$ en los contenidos de proteína, lípido, carbohidrato y energía entre las muestras F1 y F3. Se concluye que un nivel de adición de hasta un $6 \%$ de HRB en cupcake mejora el perfil nutricional del producto. Además, presenta aceptación similar al producto estándar, con buenas expectativas de comercialización.

Palabras clave: nutrimentos; fibras en la dieta; aprovechamiento integral de los alimentos. 
Cupcake adicionado de farinha de resíduos de brócolis: análise físico-química e sensorial entre crianças

\section{INTRODUÇÃO}

Aproximadamente um terço de toda a produção de alimentos mundial é desperdiçada pela população. O desperdício ocorre em toda a cadeia alimentar, desde a produção até o consumo. Apesar disso, o número de pessoas subalimentadas cresce a cada ano. Em 2016, 815 milhões de pessoas receberam alimentação insuficiente, número ainda maior que 2015 que foi de 777 milhões. Os alimentos que mais apresentam perdas são as frutas e os vegetais, principalmente de seus subprodutos, como as cascas, talos e folhas, apesar de conterem um elevado valor nutritivo. Uma alternativa para evitar o descarte seria utilizar esses alimentos como ingredientes em produtos normalmente consumidos pela população. Nyam, Lau e Tan (2013) verificaram que a adição de $5 \%$ de casca de abóbora em pão elevou sua aceitabilidade. Também, houve um aumento nos teores de fibra dietética e de compostos fenólicos, além de uma melhora na textura e na maciez do produto. Na infância, o consumo de nutrientes específicos, como vitaminas, minerais e fibras, pode colaborar para melhorar as funções cognitiva e neuronal e no controle da glicemia.

A fase escolar é caracterizada pela faixa etária de 7 a 10 anos, na qual as crianças se tornam mais independentes. Contudo os hábitos das crianças são influenciados pelo consumo alimentar da família, pelo ambiente escolar e a pela mídia. Nesse aspecto, atualmente, verifica-se uma elevada ingestão de alimentos ultraprocessados, com altos teores de sódio, açúcar e gordura. Esse tipo de dieta pode elevar a prevalência de casos de obesidade e de doenças cardiovasculares. No caso de crianças, já foi demonstrado que a ingestão diária de açúcar pode ultrapassar quase duas vezes a recomendação da Organização Mundial da Saúde (OMS), que é no máximo de 10\% do total da composição da alimentação diária. Em contrapartida, sabe-se que crianças consomem uma baixa quantidade de hortaliças (2-3 vezes/ semana) e frutas (4-6 vezes/semana), bem abaixo da recomendação ideal, que é de cinco porções diárias. Nesse contexto, destaca-se a importância do desenvolvimento de produtos com um melhor perfil nutricional para essa faixa etária, o que poderá reduzir o risco de doenças crônicas não transmissíveis futuras. 
As hortaliças pertencentes ao gênero Brassica incluem couve, couve-flor, brócolis e repolho e estão entre as 10 culturas mais importantes na economia mundial. A produção de brócolis (Brassica oleracea var. italica) ocorre em regiões de clima temperado, principalmente nas estações do outono e de inverno. Dentre os países que mais se destacam na produção do brócolis e couve-flor estão a China com 10.263.746 t/ano e a Espanha com 605.161 t/ano. Em países em desenvolvimento como o Brasil, a produção e comercialização dos brócolis vêm crescendo gradualmente, com um volume comercializado de 14.745 toneladas em 2014. Os brócolis contêm elevados teores de carotenoides, vitaminas C e E e compostos fenólicos. Além disso, é fonte de glucosinolatos, que podem reduzir o risco do desenvolvimento de certos tipos de câncer. Comumente, os resíduos dos brócolis (folhas e talos) são utilizados para o consumo animal ou descartado pelo consumidor final durante o processamento. Entretanto esses resíduos podem apresentar até seis vezes mais fibra e duas vezes mais minerais que a flor dos brócolis, que a parte mais consumida. Esse fato favorece sua adição como ingrediente em produtos alimentícios, tais como bolos, pães e biscoitos, visando ao enriquecimento nutricional.

Os produtos de panificação apresentam elevada aceitabilidade e consumo por diferentes faixas etárias. No Brasil, o setor geral de panificação (pão, bolo e biscoito) foi responsável pela exportação de 2.992 .790 kg em 2016. Os bolos se destacam já que o faturamento mundial chegou a 27 miIhões de dólares no ano de 2017. Contudo seu consumo per capita no país ainda é discreto, girando em torno de 0,16 kg/ano. Os bolos são produtos obtidos pela mistura de farinha(s), ovo e fermento podendo conter outros ingredientes e diferentes sabores. O cupcake é um tipo de bolo servido de forma individual, bem aceito por todas faixas etárias, principalmente pelo público infantil devido aos seus atributos atrativos. Além disso, esses produtos apresentam um shelf-life mais longo, assim como um potencial tecnológico para adição de novos ingredientes. Contudo, geralmente, têm elevados teores de calorias, açúcares e gorduras. Uma forma de modificar favoravelmente o perfil nutricional do cupcake é a adição de ingredientes que contenham vitaminas, minerais e fibras, como é o caso dos subprodutos de frutas e hortaliças. Após a adição dessas matérias-primas, faz-se 
Cupcake adicionado de farinha de resíduos de brócolis: análise físico-química e sensorial entre crianças

necessária a realização de testes sensoriais e físico-químicos para garantir a qualidade do produto.

Avaliações sensoriais são fundamentais para garantir a qualidade durante o processo de desenvolvimento de alimentos. No caso de produtos destinados ao público infantil, as avaliações necessitam de cuidados ainda mais específicos, já que as crianças estão em fase de crescimento e desenvolvimento. Geralmente, as crianças participam de análises sensoriais por meio de instrumentos lúdicos adequados à idade, garantindo a eficiência dos resultados. O estudo da composição físico-química deve ser também um objetivo na elaboração de novos produtos. Essas análises são utilizadas para a caracterização nutricional e tecnológica do alimento, contribuindo para a segurança alimentar e nutricional dos consumidores. Nesse contexto, o objetivo do estudo foi avaliar a aceitabilidade sensorial de cupcake adicionado de diferentes níveis de Farinha de Resíduos de Brócolis (FRB). Também, determinar a composição físico-química da formulação padrão e daquela contendo maior teor de FRB e com aceitação semelhante à padrão.

\section{MATERIAIS E MÉTODOS}

Os ingredientes foram adquiridos em supermercados do município de Guarapuava, PR. Foram utilizados talos e folhas de brócolis (Brassica oleracea var. italica), com melhor aspecto visual, consistência firme, sem imperfeições e de coloração esverdeada. Vinte quilos de talos e folhas de brócolis foram higienizados em água corrente potável, sanitizados (solução de hipoclorito de sódio, 200 ppm, por 15 minutos) e novamente higienizados em água corrente. Os talos e folhas foram extraídos de forma manual e processados. Em seguida, foram submetidas à secagem em desidratador (Pardal ${ }^{\circledR}$, Brasil) com circulação de $\operatorname{ar}\left(65^{\circ} \mathrm{C}\right)$ por 48 horas. Depois de secos, permaneceram em temperatura ambiente $\left(22^{\circ} \mathrm{C}\right)$ até total resfriamento. Os resíduos foram triturados em liquidificador doméstico (Mondial ${ }^{\circledR}$, Brasil) e passados em peneira com abertura de 32 mesh/Tyler (Bertel ${ }^{\circledR}$, Brasil) até a obtenção da FRB, que obteve um rendimento de 1,7 kg.

Foram elaboradas 5 formulações de cupcake adicionadas de diferentes níveis de FRB: F1 (0\%, padrão), F2 (3\%), F3 (6\%), F4 (9\%) e F5 (12\%). Essas 
porcentagens foram definidas por meio de testes sensoriais preliminares realizados com o produto. Além da FRB, os ingredientes utilizados nas formulações foram: farinha de trigo (F1: 22\%, F2: 19\%, F3: 16\%, F4: 13\%, F5: $10 \%)$, leite integral $(29,43 \%)$, ovos $(2 \%)$, fermento biológico fresco $(2,21 \%)$, manteiga $(1,47 \%)$, sal $(0,44 \%)$, presunto $(22,08 \%)$ e queijo muçarela $(22,08 \%)$. O cupcake foi preparado misturando-se a farinha de trigo, a FRB, o fermento, o leite, os ovos e o sal em uma batedeira doméstica (Mondial ${ }^{\circledR}$, Brasil) até completa homogeneização. Metade da massa foi disposta em formas de alumínio arredondadas (diâmetro superior de $8,5 \mathrm{~cm}$, inferior de $5 \mathrm{~cm}$ e altura de $2 \mathrm{~cm}$ ), untadas com manteiga. Em seguida, foram adicionados o presunto e o queijo, completando-se com a outra metade da massa. As formulações foram

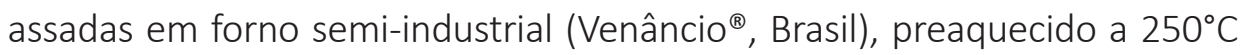
por 30 minutos. Após esse processo, permaneceram em repouso até atingirem a temperatura ambiente $\left(22^{\circ} \mathrm{C}\right)$. Os produtos foram acondicionados em recipientes plásticos hermeticamente fechados até o momento das análises.

Participaram da análise sensorial 83 julgadores não treinados, sendo crianças devidamente matriculadas em uma Escola Municipal de Guarapuava, PR, de ambos os sexos, com idade entre 7 a 10 anos. Os produtos foram submetidos à análise sensorial em uma sala da escola. Cada julgamento sensorial foi feito individualmente, sendo que o provador foi orientado pelas pesquisadoras para o preenchimento das respostas. Foram avaliados os atributos de aparência, aroma, sabor, textura e cor, por meio de uma escala hedônica facial estruturada mista de 7 pontos variando de 1 (super ruim) a 7 (super bom). Também, foram aplicadas questões de aceitação global e de intenção de compra analisadas com o uso de uma escala estruturada de 5 pontos ( 1 - desgostei muito/não compraria a 5 - gostei muito/compraria com certeza) (DUTCOSKY, 2013). Os julgadores receberam uma porção (15 g) de cada cupcake (peso médio de $50 \mathrm{~g}$ ) em pratos brancos descartáveis, codificados com números de três dígitos, de forma casualizada e balanceada, acompanhadas de um copo de água para limpeza do palato. As formulações foram oferecidas aos julgadores de forma monádica sequencial. O cálculo do IA foi realizado conforme a fórmula: $I A(\%)=A \times 100 / B$ (onde: $A=$ nota média obtida para o produto e $B=$ nota máxima dada ao produto) (TEIXEIRA; MEINERT; BARBETTA, 1987). 
Cupcake adicionado de farinha de resíduos de brócolis: análise físico-química e sensorial entre crianças

As seguintes análises físico-químicas foram realizadas em triplicata na FRB, na formulação padrão e naquela com maior nível de adição de FRB e com aceitação sensorial semelhante ao produto padrão: Umidade: determinada em estufa a $105^{\circ} \mathrm{C}$ até peso constante; Cinzas: analisadas em mufla $\left(550^{\circ} \mathrm{C}\right)$ (AOAC, 2011); Lipídio: utilizou-se o método de extração a frio (BLIGH; DYER, 1959); Proteína: avaliadas através do teor de nitrogênio total da amostra, pelo método Kjeldahl, determinado ao nível semimicro (AOAC, 2011). Utilizou-se o fator de conversão de nitrogênio para proteína de 6,25; Fibra alimentar total e insolúvel: determinadas por método enzimático (AOAC, 2011). O teor de fibra alimentar solúvel foi calculado pela diferença dos resultados de fibra alimentar total e insolúvel; Carboidrato: avaliados por cálculo teórico (por diferença) nos resultados das triplicatas, conforme a fórmula: $\%$ Carboidrato = 100 - (\% umidade + \% proteína + \% lipídio + \% cinzas + \% fibra); Valor calórico total (kcal): calculado utilizando-se os seguintes valores: lipídio (8,37 kcal/g), proteína (3,87 kcal/g) e carboidrato (4,11 kcal/g) (MERRILL; WATT, 1973). O Valor Diário de Referência (VD): calculado em relação a $46 \mathrm{~g}$ de cupcake, com base nos valores médios diários preconizados para crianças (7 a 10 anos) (DRI, 2005), resultando em: 1.933,5 kcal/dia, $130 \mathrm{~g} /$ dia de carboidrato, 26,5 $\mathrm{g} /$ dia de proteína e 26,75 g/dia de fibra alimentar.

Os dados foram analisados com auxílio do software Statgraphics Plus ${ }^{\circledR}$, versão 5.1, por meio da Análise de Variância (ANOVA). A comparação de médias foi realizada pelos testes de Tukey e t de student, avaliados com nível de 5\% de significância.

Este trabalho foi aprovado pelo Comitê de Ética em Pesquisa da Universidade Estadual do Centro-Oeste (UNICENTRO), parecer n. 2.451.570/2017. Entretanto foram considerados como critérios de exclusão os seguintes fatores: ter alergia a algum ingrediente utilizado na elaboração do cupcake ou não entregar o Termo de Consentimento Livre e Esclarecido (TCLE) assinado pelo responsável legal.

\section{RESULTADOS E DISCUSSÃO}

Os resultados da análise sensorial do cupcake adicionado de diferentes níveis FRB estão descritos na Tabela 1. 
Tabela 1 - Escores sensoriais (média desvio-padrão) e Índice de Aceitabilidade (IA) do cupcake com adição de diferentes níveis de Farinha de Resíduos de Brócolis (FRB)

\begin{tabular}{|c|c|c|c|c|c|}
\hline Parâmetros & F1 & F2 & F3 & F4 & F5 \\
\hline Aparência & $6,03 \pm 0,86^{a}$ & $5,45 \pm 1,00^{\mathrm{ab}}$ & $5,50 \pm 1,04^{\mathrm{ab}}$ & $5,00 \pm 1,25^{b}$ & $4,95 \pm 1,49^{b}$ \\
\hline IA (\%) & 86,14 & 77,86 & 78,57 & 71,43 & 70,71 \\
\hline Aroma & $5,76 \pm 0,94^{a}$ & $5,37 \pm 0,98^{\mathrm{ab}}$ & $5,25 \pm 1,03^{\mathrm{abc}}$ & $5,09 \pm 1,22^{b c}$ & $4,78 \pm 1,25^{c}$ \\
\hline IA (\%) & 82,29 & 76,71 & 75,00 & 72,71 & 68,28 \\
\hline Sabor & $6,18 \pm 0,84^{\mathrm{a}}$ & $5,79 \pm 1,15^{\mathrm{a}}$ & $5,57 \pm 1,08^{a b}$ & $4,99 \pm 1,43^{b c}$ & $4,88 \pm 1,48^{c}$ \\
\hline IA (\%) & 88,28 & 82,71 & 79,57 & 71,28 & 69,71 \\
\hline Textura & $5,83 \pm 0,92^{\mathrm{a}}$ & $5,59 \pm 0,94^{a b}$ & $5,47 \pm 1,0^{\mathrm{abc}}$ & $5,16 \pm 1,22^{b c}$ & $5,00 \pm 1,23^{c}$ \\
\hline IA (\%) & 83,28 & 79,85 & 78,14 & 73,71 & 71,43 \\
\hline Cor & $5,86 \pm 1,11^{\mathrm{a}}$ & $5,34 \pm 1,06^{\mathrm{ab}}$ & $5,33 \pm 1,05^{a b}$ & $5,04 \pm 1,15^{b c}$ & $4,72 \pm 1,28^{c}$ \\
\hline IA (\%) & 83,71 & 76,28 & 76,14 & 72,00 & 67,43 \\
\hline $\begin{array}{c}\text { Aceitação } \\
\text { global }\end{array}$ & $4,56 \pm 0,56^{a}$ & $4,33 \pm 0,62^{a}$ & $4,23 \pm 0,55^{a b}$ & $3,88 \pm 0,86^{b c}$ & $3,73 \pm 0,84^{c}$ \\
\hline IA (\%) & 91,20 & 86,60 & 84,60 & 77,60 & 74,60 \\
\hline $\begin{array}{l}\text { Intenção de } \\
\text { compra }\end{array}$ & $4,63 \pm 0,67^{a}$ & $4,22 \pm 0,90^{a}$ & $4,23 \pm 0,77^{\mathrm{a}}$ & $3,76 \pm 1,08^{b}$ & $3,66 \pm 1,17^{b}$ \\
\hline
\end{tabular}

Legenda: Letras sobrescritas distintas na linha indicam diferença significativa pelo teste de Tukey (p<0,05); Adição de FRB: F1: 0\%; F2: 3\%; F3: 6\%; F4: 9\%; F5: 12\%.

Fonte: Autoria própria.

Maiores notas $(p<0,05)$ para a aparência foram verificadas para F1 quando comparadas a F4 e F5. Não houve diferença estatística entre as demais amostras $(p>0,05)$. Para os atributos aroma e textura, houve também maior aceitabilidade para F1 em relação à F4 e F5. Além disso, F2 foi também mais aceita que F5, sem diferença entre as outras formulações. As amostras F1 e F2 foram mais aceitas que F4 e F5 nas avaliações de sabor e aceitação global, F3 apresentou também maior aceitabilidade que F5, enquanto F4 e F5 não deferiram significativamente $(p>0,05)$ nessas características. De 
Cupcake adicionado de farinha de resíduos de brócolis: análise físico-química e sensorial entre crianças

forma similar, as formulações F4 e F5 foram menos aceitas ( $p<0,05)$ que F1 no atributo de cor, F5 recebeu também menores notas quando comparada a F2 e F3. Maior intenção de compra foi constatada para as formulações F1, F2 e F3, enquanto F4 e F5 não deferiram entre si. Assim, é possível concluir que a adição de níveis superiores a 6\% de FRB reduzem a aceitabilidade de cupcake. Resultados similares foram verificados em bolo adicionado de 2,5\%, $5 \%$ e 7,5\% de folhas de brócolis (DRABIŃSKA et al., 2018) e em tortilhas contendo farinha da flor e folha de brócolis (2\%, 4\% e 8\%) (DURÁN et al., 2014).

Na presente pesquisa, a adição de FRB promoveu uma coloração esverdeada no cupcake, que foi mais intensa nas formulações com maiores teores do resíduo. Esse efeito está relacionado à elevada presença de clorofila na folha $\left(4.478 \mu \mathrm{g} \cdot \mathrm{g}^{-1}\right.$ ) e no talo de brócolis (144 $\mu \mathrm{g} \cdot \mathrm{g}^{-1}$ ) (LIU et al., 2018). Além disso, a adição de subprodutos de vegetais que tenham alto teor de fibra pode diminuir o volume e aumentar a firmeza da massa (ELLEUCH et al., 2011), o que pode explicar as menores notas para a textura nas amostras F4 e F5. Os resíduos de brócolis contêm também elevado teor de compostos fenólicos (3,8 mg.GAE.g ${ }^{-1}$ ) (THOMAS et al., 2018), o que causa um sabor adstringente no produto (LESSCHAEVE; NOBLE, 2005). Essa característica explica as menores notas para o sabor obtidas nos produtos com maiores teores de FRB. Durante a preparação do cupcake, verificou-se um aspecto mais quebradiço para as formulações com maior concentração de FRB (F4 e F5), já que as cascas de vegetais são isentas de glúten. O glúten é um complexo proteico presente no trigo, formado pelas proteínas gliadina e glutenina, que são responsáveis pela elasticidade da massa (LAMACCHIA et al., 2014). Resultados similares foram verificados em bolos adicionados de $10 \%$ de farinha casca de abóbora (NYAM; LAU; TAN, 2013). Apesar das alterações tecnológicas observadas no cupcake após a adição de FRB, os resultados sensoriais são positivos. Isso colabora para que as crianças tenham um consumo alimentar mais saudável, especialmente pela maior concentração de fibra no produto. A ingestão adequada de fibras está associada à redução do risco do desenvolvimento de doenças crônicas não transmissíveis na infância, como a obesidade e a diabetes mellitus (KRANZ et al., 2012). Na Figura 1 estão apresentadas as formulações de cupcake adicionadas de FRB. 
Figura 1 - Formulações de cupcake (vista superior) adicionadas de Farinha de Resíduos de Brócolis

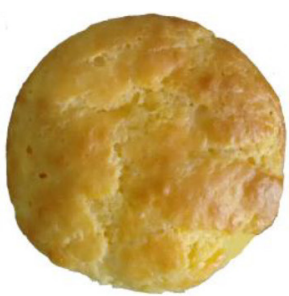

F1

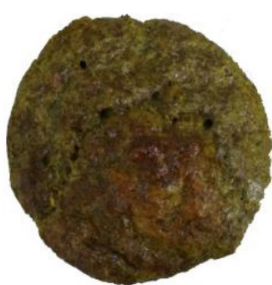

F2

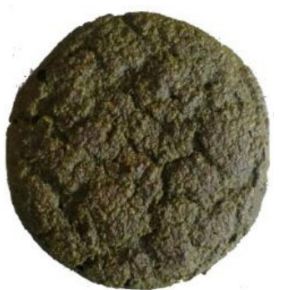

F3

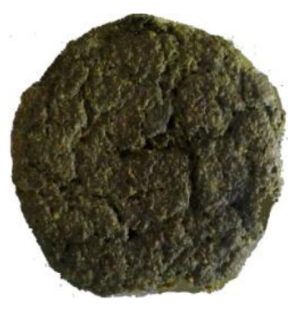

F4

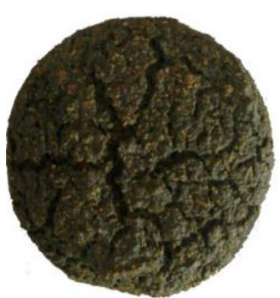

F5

Legenda: F1 (0\%), F2 (3\%), F3 (6\%), F4 (9\%) e F5 (12\%).

Fonte: Autoria própria.

As formulações apresentaram IA acima de $70 \%$ para todas as avaliações, com exceção de F5 para os atributos aroma, sabor e cor. Segundo Teixeira, Meinert e Barbetta (1987), um produto com IA igual ou superior a $70 \%$ pode ser classificado como bem aceito pelos consumidores. Efeitos similares foram relatados por Ribeiro et al. (2015) em cookie com adição de até $10 \%(\mid A=74 \%)$ de farinha de resíduos de couve-flor. A distribuição dos julgadores pelos valores hedônicos obtidos no teste sensorial está apresentada na Figura 2. 
Cupcake adicionado de farinha de resíduos de brócolis: análise físico-química e sensorial entre crianças

Figura 2 - Distribuição dos julgadores pelos valores hedônicos obtidos no teste de aceitabilidade das formulações de cupcake adicionadas de diferentes níveis de Farinha de Resíduos de Brócolis
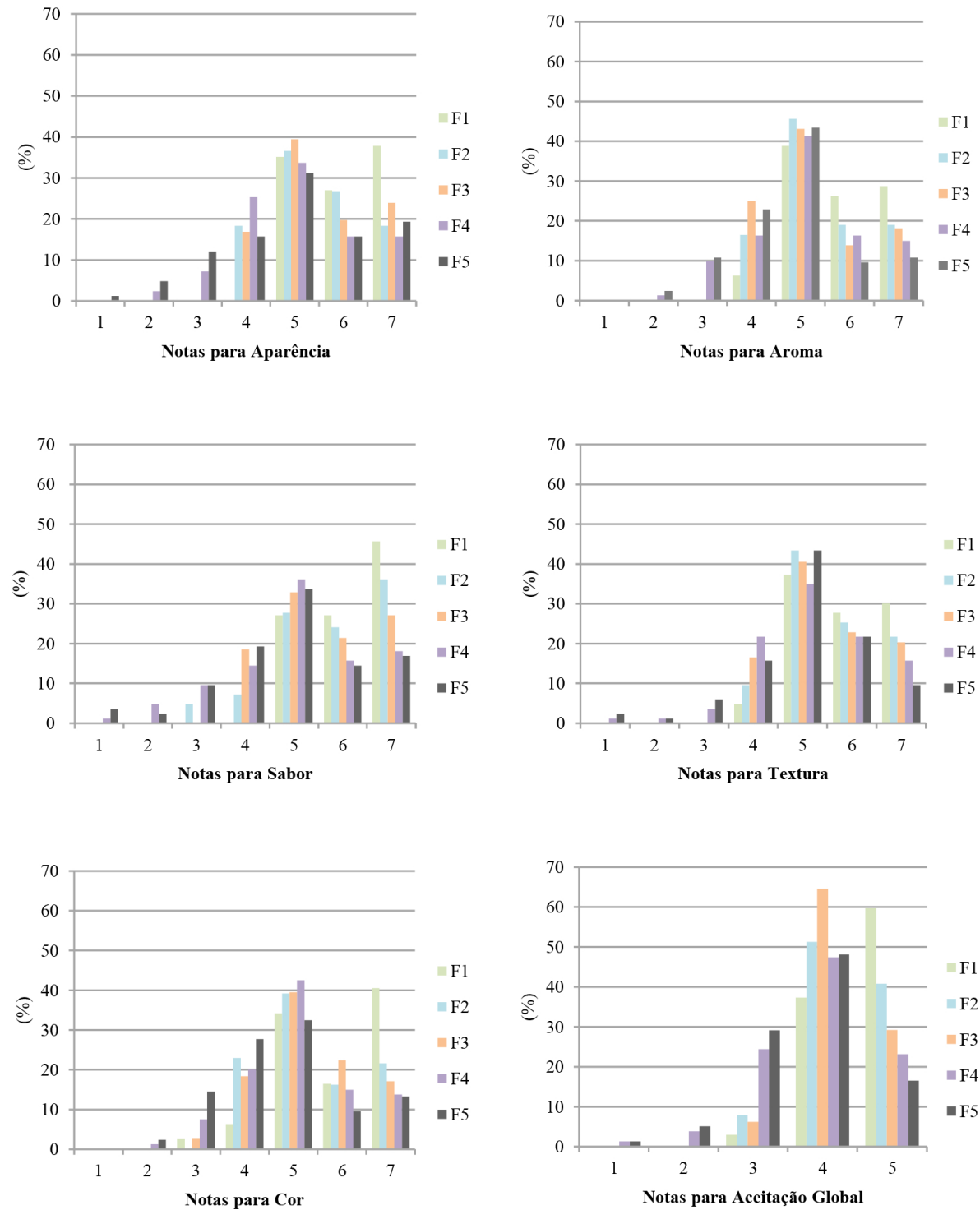

Legenda: 0\% (F1), 3\% (F2), 6\% (F3), 9\% (F4) e 12\% (F5).

Fonte: Autoria própria. 
Maior porcentagem de julgamentos foi obtida para as notas $\geq 5$ (bom) para os atributos e $\geq 4$ (gostei) para aceitação global, corroborando com a literatura (DRABIŃSKA et al., 2018). Considerando que a amostra F3 foi aquela com maior teor de FRB e com aceitação sensorial similar à padrão em todas as avaliações (Tabela 1), ela foi selecionada para fins de comparação físico-química juntamente da formulação padrão (F1) (Tabela 2).

Tabela 2 - Composição físico-química média ( \pm desvio-padrão) da Farinha de Resíduos de Brócolis (FRB), do cupcake sem adição de FRB (F1) e com adição de 6\% (F3) de FRB

\begin{tabular}{|c|c|c|c|c|c|}
\hline Parâmetro & FRB & F1 & $\begin{array}{l}\text { VD } \\
(\%)^{*}\end{array}$ & F3 & $\begin{array}{l}\text { VD } \\
(\%)^{*}\end{array}$ \\
\hline $\begin{array}{l}\text { Umidade } \\
\left({\left.\mathrm{g} .100 \mathrm{~g}^{-1}\right)}\right.\end{array}$ & $7,87 \pm 0,05$ & $56,50 \pm 0,03^{a}$ & ND & $54,22 \pm 0,05^{b}$ & ND \\
\hline $\begin{array}{c}\text { Cinzas } \\
\left(\mathrm{g} .100 \mathrm{~g}^{-1}\right)\end{array}$ & $8,14 \pm 0,07$ & $2,83 \pm 0,04^{b}$ & ND & $3,63 \pm 0,02^{a}$ & ND \\
\hline $\begin{array}{l}\text { Proteína } \\
\left(\mathrm{g} .100 \mathrm{~g}^{-1}\right)\end{array}$ & $17,27 \pm 0,08$ & $13,59 \pm 0,03^{\mathrm{a}}$ & 23,59 & $13,87 \pm 0,09^{a}$ & 24,07 \\
\hline $\begin{array}{l}\text { Lipídio } \\
\left({\left.\mathrm{g} .100 \mathrm{~g}^{-1}\right)}\right.\end{array}$ & $4,27 \pm 0,04$ & $9,10 \pm 0,05^{a}$ & ND & $9,44 \pm 0,06^{a}$ & ND \\
\hline $\begin{array}{l}\text { Carboidrato } \\
\left(\mathrm{g} .100 \mathrm{~g}^{-1}\right)^{* *}\end{array}$ & $62,44 \pm 0,23$ & $17,98 \pm 0,13^{\mathrm{a}}$ & 6,36 & $18,84 \pm 0,55^{a}$ & 6,66 \\
\hline 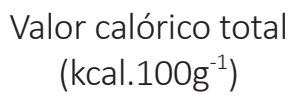 & $359,25 \pm 0,96$ & $202,69 \pm 3,78^{a}$ & 4,82 & $210,08 \pm 4,8 w 7^{a}$ & 4,99 \\
\hline $\begin{array}{l}\text { Fibra solúvel } \\
\left(\mathrm{g} .100 \mathrm{~g}^{-1}\right)^{* * *}\end{array}$ & $3,33 \pm 0,16$ & $0,02 \pm 0,09^{b}$ & ND & $0,21 \pm 0,04^{a}$ & ND \\
\hline $\begin{array}{l}\text { Fibra insolúvel } \\
\left(\mathrm{g}^{\prime} 100 \mathrm{~g}^{-1}\right)^{* * *}\end{array}$ & $24,98 \pm 0,12$ & $0,52 \pm 0,11^{b}$ & ND & $1,88 \pm 0,12^{a}$ & ND \\
\hline $\begin{array}{l}\text { Fibra total } \\
\left(\mathrm{g} .100 \mathrm{~g}^{-1}\right)^{* * *}\end{array}$ & $28,30 \pm 0,10$ & $0,54 \pm 0,09^{b}$ & 0,92 & $2,09 \pm 0,13^{a}$ & 3,59 \\
\hline
\end{tabular}

Legenda: Letras sobrescritas distintas na linha indicam diferença significativa pelo teste de $t$ de student $(p<0,05)$; *Valor Diário de Referência (VD): nutrientes avaliados pela média da DRI (2005), com base numa dieta de 1.933,5 kcal/dia e porção média de 46 gramas de produto; Valores apresentados em base úmida; ${ }^{* *}$ Inclui fibra alimentar; ${ }^{* * *}$ Fibra alimentar; ND: não disponível.

Fonte: Autoria própria. 
Cupcake adicionado de farinha de resíduos de brócolis: análise físico-química e sensorial entre crianças

Valores inferiores ao presente trabalho para a FRB foram verificados na literatura para proteína $\left(15,6 \mathrm{~g} \cdot 100 \mathrm{~g}^{-1}\right)$, lipídio $\left(2,4 \mathrm{~g} \cdot 100 \mathrm{~g}^{-1}\right)$ e carboidrato $\left(34,8\right.$ g. $\left.100 \mathrm{~g}^{-1}\right)$; e apresentaram-se valores superiores para umidade $(14,9$ g. $\left.100 \mathrm{~g}^{-1}\right)$ e cinzas $\left(12,9 \mathrm{~g}^{1} 100 \mathrm{~g}^{-1}\right)$ (MALUCELLI et al., 2009). Alguns fatores podem influenciar no teor nutricional da FRB, como o tempo e a temperatura referentes ao processo de obtenção da farinha, a qualidade do solo, o período do ano e as condições climáticas de plantio, assim como a espessura dos resíduos utilizados para as análises (CHISTÉ; COHEN, 2011; EMBRAPA, 2015; ANDRADE et al., 2018). O teor de cinzas presente na FRB demonstra a elevada concentração de minerais, principalmente o cálcio $\left(49,8 \mathrm{mg} \cdot 100 \mathrm{~g}^{-1}\right)$ e o potássio (325 mg.100g ${ }^{-1}$ ) (USDA, 2018). Desse modo, a FRB colabora para melhorar o valor nutricional das preparações. Ainda, tem um elevado teor de fibra, que contribui para melhorar o trânsito intestinal, reduzir o colesterol sérico e o risco de desenvolvimento de doenças cardiovasculares (LAMBEAU; MCRORIE, 2017). A FRB apresentou teor de umidade conforme o recomendado pela RDC n. 263 para farinhas, que é de até 15\% (BRASIL, 2005). Um baixo teor de umidade colabora para aumentar o shelf-life do produto, já que a maioria dos micro-organismos depende de altos valores de atividade água para se desenvolver (FERRARI-FELISBERTO et al., 2015).

Teor de umidade mais elevado $(p<0,05)$ foi constatado em $F 1$, o que se deve a maior quantidade de umidade na farinha de trigo $\left(10,59 \mathrm{~g} \cdot 100 \mathrm{~g}^{-1}\right)$ (USDA, 2018), quando comparada à FRB (Tabela 2). Contudo F3 apresentou maior conteúdo de cinzas, já que farinha de trigo contém menor concentração de minerais $\left(4,33\right.$ g.100 $\mathrm{g}^{-1}$ ) (USDA, 2018) que a FRB. Não houve diferença significativa $(p>0,05)$ entre os teores de proteína, lipídio, carboidrato e energia de F1 e F3. O cupcake adicionado de 6\% de FRB apresentou maior quantidade $(p<0,05)$ de fibra solúvel, insolúvel e total que a formulação padrão, com um aumento de $287 \%$ em relação à $\mathrm{F} 1$. Esse resultado está relacionado ao elevado teor de fibras presente na $\operatorname{FRB}\left(28,30 \mathrm{~g}^{\circ} 100 \mathrm{~g}^{-1}\right)$, bem superior ao encontrado na farinha de trigo $\left(2,7 \mathrm{~g} \cdot 100 \mathrm{~g}^{-1}\right)($ USDA, 2018).

\section{CONCLUSÕES}

Um nível de adição de até 6\% de farinha de resíduos de brócolis em cupcakes é bem aceito pelas crianças, obtendo-se aceitação sensorial similar 
ao produto padrão. Além disso, melhora o perfil nutricional do produto, aumentando os teores de cinzas e de fibra alimentar. Dessa forma, a farinha de resíduo de brócolis pode ser considerada um potencial ingrediente para adição em cupcake e produtos similares, podendo ser oferecida ao público infantil com altas expectativas de comercialização.

\section{AGRADECIMENTOS}

Os autores agradecem à Fundação Araucária de Apoio à Pesquisa do Estado do Paraná, pela concessão da bolsa ao Programa Institucional de Bolsas de Extensão Universitária, PIBEX/UNICENTRO.

\section{REFERÊNCIAS}

ANDRADE, B. A.; PERIUS, D. B.; MATTOS, N. V.; LUVIELMO M. M. Produção de farinha de banana verde (musa spp.) para aplicação em pão de trigo integral. Brazilian Journal of Food Technology, Campinas, v. 21, n. 1, p. 10-1, nov. 2018.

ASSOCIATION OF OFFICIAL AGRICULTURAL CHEMISTS [AOAC]. Official Methods of Analysis of AOAC International. 18. ed. Arlington: AOAC, 2011.

BLIGH, E. G.; DYER, W. J. A rapid method of total lipid extraction and purification. Canadian Journal of Biochemistry and Physiology, Ottawa, v. 37, n. 8, p. 911-7, fev. 1959.

BRASIL. Ministério da Saúde. Agência Nacional de Vigilância Sanitária (ANVISA). Resolução RDC n. 263, de 22 de setembro de 2005. Regulamento técnico para produtos de cereais, amidos, farinhas e farelos. Diário Oficial União, Brasília-DF, 22 set. 2005.

CHISTÉ, R. C.; COHEN, K. D. E. O. Influência da fermentação na qualidade da farinha de mandioca do grupo d'água. Acta Amazonica, Manaus, v. 41, n. 2, p. 279-84, nov. 2011.

DIETARY REFERENCE INTAKES [DRI]. Dietary Reference Intakes for energy, carbohydrate, fiber, fat, fatty acids, cholesterol, protein and amino acids. Washington: National Academy Press, 2005. 
Cupcake adicionado de farinha de resíduos de brócolis: análise físico-química e sensorial entre crianças

DRABIŃSKA, N.; CISKA, E.; SZMATOWICZ, B.; KRUPA-KOZAK, U. Broccoli by-products improve the nutraceutical potential of gluten-free mini sponge cakes. Food Chemistry, Barking, v. 267, n. 1, p. 170-7, nov. 2018.

DURÁN, V.; SOTO, A.; BARRAGAN, H. B.; ALBOREZ, A. M. Physicochemical, nutritional and sensory properties of deep-fat fried fortified tortilla chips with broccoli (Brassica oleracea L. convar. italica Plenck) flour. Journal of Food and Nutrition Research, Balsta, v. 53, n. 4, p. 313-24, set. 2014.

DUTCOSKY, S. D. Análise sensorial de alimentos. 4. ed. Curitiba: Champagnat, 2013. ELLEUCH, M.; BEDIGIAN, D.; ROISEUX, O.; BESBES, S.; BLECKER, C.; ATTIA, H. Dietary fibre and fibre-rich by-products of food processing: characterization, technological functionality and commercial applications - a review. Food Chemistry, Barking, v. 124, n. 2, p. 411-21, jan. 2011.

EMPRESA BRASILEIRA DE PESQUISA AGROPECUÁRIA [EMBRAPA]. A cultura dos brócolis. Brasília: Coleção Plantar, 2015.

FERRARI-FELISBERTO, M. H.; WAHANICK, A. L.; GOMES-RUFFI, C. R.; CLERICI, M. T. P. S.; CHANG, Y. K.; STEEL, C. S. Steel Use of chia (Salvia hispanica L.) mucilage gel to reduce fat in pound cakes. LWT - Food Science and Technology, London, v. 63, n. 2, p. 1049-55, out. 2015.

KRANZ S.; BRAUCHLA, M.; SLAVIN, J. L.; MILLER, K. B. What do we know about dietary fiber intake in children and health? The effects of fiber intake on constipation, obesity, and diabetes in children. Advances in Nutrition, Oxford, v. 3, n. 1, p. 47-53, jan. 2012.

LAMACCHIA, C.; CAMARCA, A.; PICASCIA, S.; DI LUCCIA, A.; GIANFRANNI, C. Cerealbased gluten-free food: How to reconcile nutritional and technological properties of wheat proteins with safety for celiac disease patients. Nutrients, London, v. 6, n. 2, p. 575-90, jan. 2014.

LAMBEAU, K. V.; MCRORIE, J. W. Fiber supplements and clinically proven health benefits: how to recognize and recommend an effective fiber therapy. Journal of the American Association Nurse Practitioners, Chicago, v. 29, n. 4, p. 216-23, abr. 2017.

LESSCHAEVE, I.; NOBLE, A. C. Polyphenols: factors influencing their sensory properties and their effects on food and beverage preferences. American Journal of Clinical Nutrition, Bethesda, v. 81, n. 1, p. 330-5, jan. 2005. 
LIU, M.; ZHANG, L.; SER, S. L.; CUMMING, J. R.; KU, K. M. Comparative phytonutrient analysis of broccoli by-Products: the potentials for broccoli by-product utilization. Molecules, Basel, v. 23, n. 4, p. 1-18, abr. 2018.

MALUCELLI, M.; NOVELLO, D.; ANDO, N.; ALMEIDA, J. M.; FREITAS, A. R. Evaluation and nutritional composition of traditional gnocchi enriched with broccoli residue flour (brassica oleracea var. Italic). Alimentos e Nutrição, Araraquara, v. 20, n. 4, p. 553-60, out./dez. 2009.

MERRILL, A. L.; WATT, B. K. Energy values of foods: basis and derivation. 74. ed. Washington: USDA, 1973. [Agricultural Handbook].

NYAM, K.; LAU, M.; TAN C. P. Fiber from pumpkin (cucurbita pepo L.) seeds and rinds: physic-chemical properties, antioxidant capacity and application as bakery product ingredients. Malaysian Journal of Nutrition, Serdang, v. 19, n. 1, p. 99-9, abr. 2013.

RIBEIRO T. C.; ABREU, J. P.; FREITAS, J. M. C.; PUMAR, M.; TEODORO, A. J. Substitution of wheat flour with cauliflower flour in bakery products: effects on chemical, physical, antioxidant properties and sensory analyses. International Food Research Journal, Essex, v. 22, n. 2, p. 532-8, set. 2015.

TEIXEIRA, E.; MEINERT, E. M.; BARBETTA, P. A. Análise sensorial de alimentos. Florianópolis: Editora UFSC, 1987.

THOMAS, M.; BADR, A.; DESJARDINS, Y.; GOSSELIN, A.; ANGERS, P. Characterization of industrial broccoli discards (brassica oleracea var. italica) for their glucosinolate, polyphenol and flavonoid contents using UPLC MS/MS and spectrophotometric methods. Food Chemistry, Barking, v. 245, n. 1, p. 1204-11, abr. 2018.

UNITED STATES DEPARTAMENT OF AGRICULTURE [USDA]. Full Report (all nutrients): 20082. USDA Food Composition Databases, Washington, 2018. Disponível em: https://ndb.nal.usda.gov/ndb. Acesso em: 20 abr. 2018. 